\title{
Seldom Alliance of Complex Compound Odontomas in Mixed Dentition!
}

\author{
Neeta S Padmawar ${ }^{1}$, Viddyasagar P Mopagar ${ }^{2}$, Vinay H Vadvadgi ${ }^{3}$, Meghna Padubidri ${ }^{4}$, Sumit Gunjal ${ }^{5}$, Sourabh R Joshi ${ }^{6}$
}

\begin{abstract}
Background: Odontomas are common odontogenic tumors and generally appear as small, solitary, or multiple radiopaque lesions found on routine radiographic examinations. They are malformations of dental tissues and may interfere with the eruption of permanent tooth. This lesion occurs because of the developmental disturbances where dental components are laid down in a disorganized manner, due to the failure of normal morphodifferentiation.

Case description: A 9-year-old boy reported to the Outpatient Department of Pediatric and Preventive Dentistry with the chief complaint of missing lower anteriors. Orthopantomogram revealed congenitally missing both lower lateral incisors and multiple pearl-like radiopacities in the association of mesial root of primary mandibular right first molar. Extraction of carious primary first molar under local anesthesia followed by the removal of multiple pearl-like structures were sent for histopathological examination.

Discussion: Odontomas are common odontogenic origin benign tumor. Clinically, odontoma may result in over-retention of deciduous teeth, non-eruption of permanent teeth, pain, expansion of the cortical bone, and displaced tooth. Prognosis is favorable and relapse is rare but in our case odontoma recurred but it did not hamper the eruption of permanent premolar.

Conclusion: Early diagnosis prevents further complications and reduces psychological and financial burden.

Keywords: Delayed eruption, Impacted tooth, Mixed dentition, Odontoma, Primary tooth.

International Journal of Experimental Dental Science (2020): 10.5005/jp-journals-10029-1200
\end{abstract}

\section{BACKGROUND}

Odontomas or hamartomas have odontogenic origin but having limited growth prospective. They are the most common type of odontogenic tumors reported.

In literature, they are divided as central (intraosseous) odontoma, peripheral (extraosseous or soft tissue) odontoma, and erupted odontoma clinically, though histologically they can be compound (tooth-like structures) or complex (mixture of odontogenic tissues without dental organization). ${ }^{1}$

Exact etiology is not yet known but it may occur due to trauma to primary dentition or inflammatory or infectious in origin. It is suggested that hyperstimulation of odontoblast and changes in genetic components are responsible for dental development that may cause development of odontoma. ${ }^{2}$

They may present as a one of the clinical manifestations of syndromes, such as basal cell nevus syndrome, Gardner syndrome, familial colonic adenomatosis, Tangier disease, or Herrmann syndrome. ${ }^{3}$

Though they are benign in nature but problems, such as disturbance in tooth eruption-impaction, delayed eruption, or retention of primary teeth, may be caused by odontomas. ${ }^{1}$

Conservative surgical excision is the treatment of choice. Compound and complex odontomas are well encapsulated and easily enucleated from the surrounding bone.

This case report highlights the importance of early diagnosis and management to ensure better prognosis.

\section{Case Description}

A 9-year-old boy reported to the Outpatient Department of Pediatric and Preventive Dentistry with the chief complaint of missing teeth in lower anterior region. Intraoral examination
1,2,4-6 Department of Pediatric and Preventive Dentistry, Rural Dental College, Loni, Ahmednagar, Maharashtra, India

${ }^{3}$ Department of Periodontology, Rural Dental College, Loni, Ahmednagar, Maharashtra, India

Corresponding Author: Neeta S Padmawar, Department of Pediatric and Preventive Dentistry, Rural Dental College, Loni, Ahmednagar, Maharashtra, India, Phone: +91 9923403377, e-mail: opneeta23@ gmail.com

How to cite this article: Padmawar NS, Mopagar VP, Vadvadgi VH, et al. Seldom Alliance of Complex Compound Odontomas in Mixed Dentition! Int J Experiment Dent Sci 2020;9(2):70-72.

Source of support: Nil

Conflict of interest: None

showed the presence of mixed dentition with missing mandibular permanent both central incisors. Along with this, carious \# 54, 64, 65, 42, 84 were present (Figs 1 and 2). Orthopantomogram (OPG) was advised, which showed congenitally missing \# 32, 42 and multiple radiopacities in association with 84 region above the tooth bud of 44 which do not show resemblance to morphology of tooth (Fig. 3). Provisional diagnosis of odontoma was performed. Medical history was not contributory and hematological investigation was not contributory. After parents' consent, extraction of 84 under local anesthesia ( $2 \%$ lignocaine with adrenaline, 1:80,000; Lignox ${ }^{\circledR}$ ) using inferior alveolar nerve block and buccal infiltration. After extraction of 84, all odontomas were removed through the socket of 84 with spoon excavator \# EX 131(GDC) without damaging the follicle of developing follicle of 44 (Fig. 4). Complete removal of odontoma was confirmed with intra-oral periapical radiograph (IOPA) (Fig. 5). Followed by irrigation of $10 \mathrm{~mL}$ tincture iodine dilute with saline (2:1), pack was given and post-extraction instructions 




Fig. 1: Intraoral examination reveals mixed dentition with missing 42 , 32 and carious 41,84

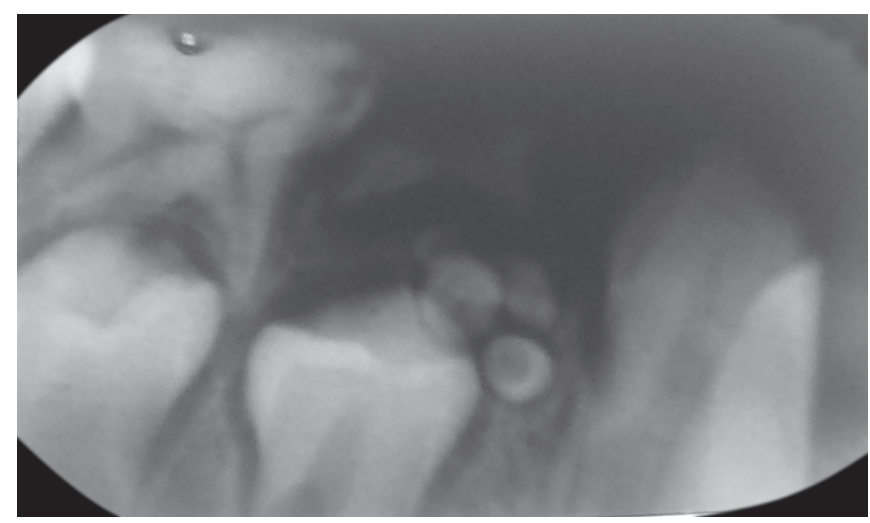

Fig. 3: Intraoral radiograph showing multiple pearl-like radiopacities in association with mesial root of 84

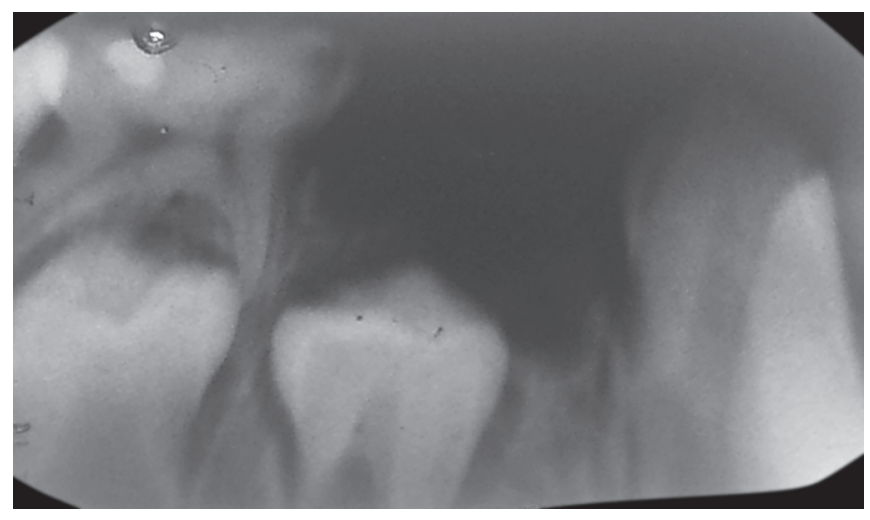

Fig. 5: Immediate postoperative IOPA of 84 region

and care were explained to patient and parent. Anti-inflammatory drug was prescribed and the patient was recalled after 24 hours, and a week for follow-up. Patient was kept under follow-up. All specimens were sent for histopathological examination. After 6-month follow-up, recurrence of odontoma was observed but it was positioned at cervical third of permanent right first premolar (Fig. 6). Due to its position near to permanent tooth, removal was not attempted and the patient was kept under further follow-up till the eruption of 44 (Fig. 7). Parents were not willing for the removal of recurred odontoma.

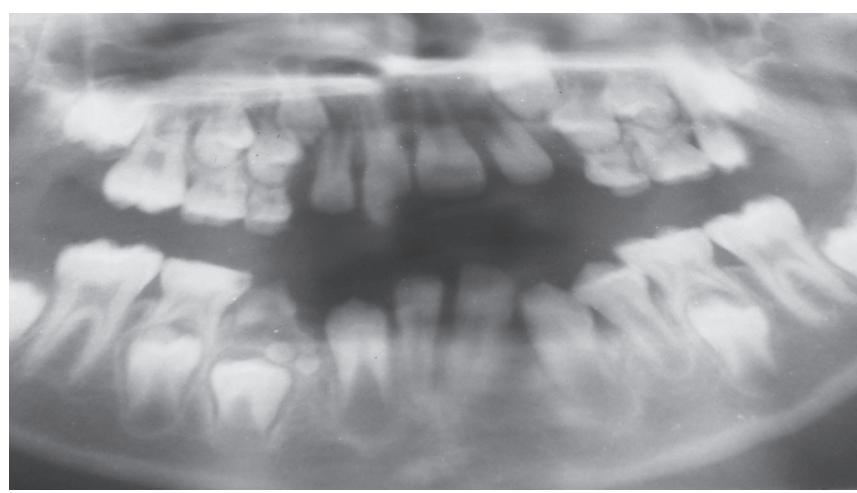

Fig. 2: Orthopantomogram showing congenitally missing 42, 32 and multiple radiopacities with 84

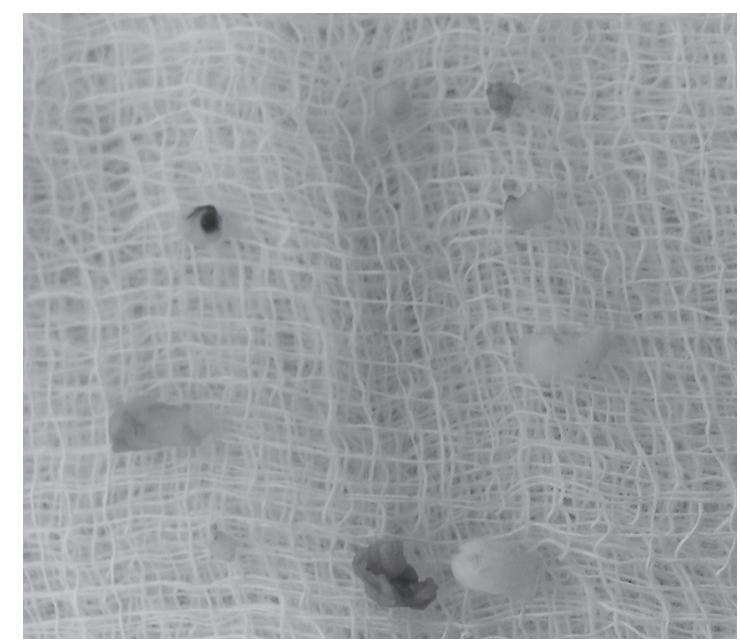

Fig. 4: Total 9, 0.5 to $1 \mathrm{~cm}$ diameter-sized pearl-like structures removed

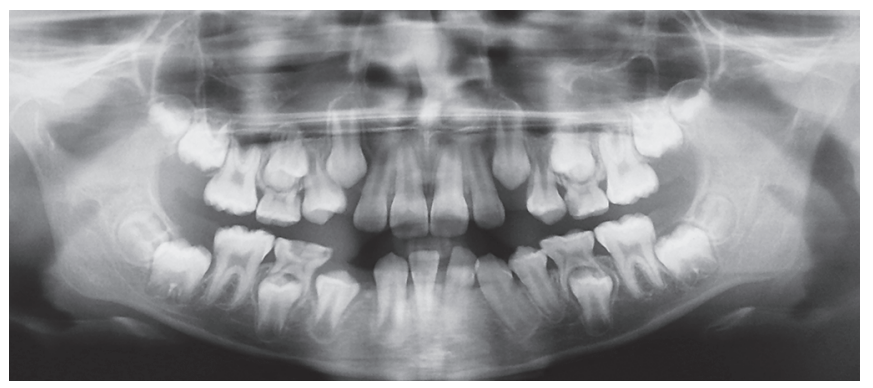

Fig. 6: Follow-up OPG showing recurred radiopacity in the association of follicle of 44

\section{Histopathological Examination}

Histological examination showed the presence of enamel, dentinal tubules, and pulp-like cells but they were arranged randomly confirming the diagnosis of complex odontoma (Fig. 8).

\section{Discussion}

Odontomas are common odontogenic origin benign tumor. Generally clinically symptomless and diagnosed in or before second 




Fig. 7: On follow-up after 1 year, intraoral examination shows erupted 44

decade of life. Frequently they are associated with impacted or over-retained tooth. ${ }^{4,5}$

In our case reported, the incidence age of odontoma is consistent with the literature.

$\mathrm{A} / \mathrm{c}$ to WHO, odontomas are classified as (1) complex odontoma-where dental tissues are well formed but less orderly arranged. (2) Compound odontoma-where all dental tissues are more orderly arranged and resembles tooth structure.

In our case, histological examination revealed less organized dental tissues which confirmed the diagnosis of complex odontoma.

If lesion is composed of more than one tissue, it is known as composite odontoma.

Further can be classified as:

- Complex composite odontoma.

- Compound composite odontoma.

In our case, histological more than one tissue was seen and was not orderly arranged, so the diagnosis was complex composite odontoma made.

Clinically, odontoma may result in over-retention of deciduous teeth, non-eruption of permanent teeth, pain, expansion of the cortical bone, and displaced tooth. But in our case, no clinical signs and symptoms were present but as diagnosis was made only on OPG. Due to early diagnosis, we could prevent the complications, such as over-retention of primary tooth and unerupted permanent tooth. ${ }^{6}$

Radiographically, depending on calcification, three stages of development of odontoma are in the first stage the lesion appears radio-lucid (due to lack of calcification of dental tissues), the intermediate stage is characterized by partial calcification, and in the final stage the odontoma appears radiopaque and surrounded by a radio-lucid halo. ${ }^{7}$

$\mathrm{A} / \mathrm{C}$ to literature, surgical extraction or extraction with removal of any associated soft tissue should be carried out to prevent complications like interference with the eruption of permanent tooth or the occurrence of dentigerous cyst.

Extraction of associated mandibular primary first molar along with removal of odontoma was carried out under local anesthesia.

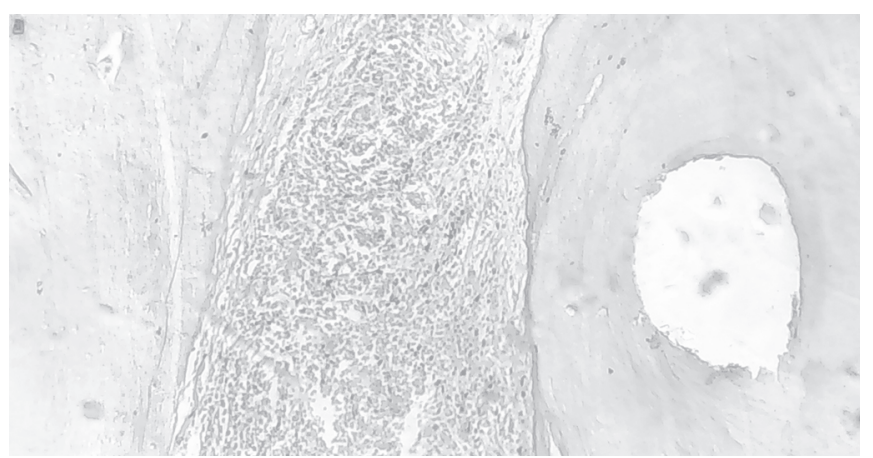

Fig. 8: Histopathologically, hematoxylin and eosin staining shows randomly arranged enamel, dentin, and fibroblast-like cells

Prognosis is favorable and relapse is rare but risk of relapse is more when nucleation is carried out in the first stage of calcification., ${ }^{7,8}$

In our case, though intermediate stage of calcification was identified radiographically but odontoma recurred but it did not hamper the eruption of permanent premolar.

\section{Conclusion}

Odontoma is the most commonly reported odontogenic tumor, mainly in the second decade of life. But it can occur in primary dentition which can result in the genesis of complications. Early diagnosis and ministrations can prevent further complications.

\section{References}

1. Kramer IRH, Pindborg JJ, Shear M. Histological typing of odontogenic tumours. WHO International histological classification of tumours. Berlin: Springer-Verlag. WHO; 1992.

2. Owens B, Schuman N, Mincer H, et al. Dental odontomas: a retrospective study of 104 cases. J Clin Pediatr Dent 1997;21(3): 261-264.

3. Sánchez OH, Berrocal ML, González JM. Meta-analysis of the epidemiology and clinical manifestations of odontomas. Med Oral Patol Oral Cir Bucal 2008;13(11):730-734.

4. Neville BW, Damm DD, Allen CM, et al. Oral and Maxillofacial Pathology. Philadelphia: Saunders; 1995. pp. 531-533.

5. Cawson RA, Binnie WH, Eveson JW. Color Atlas of Oral Disease. Clinical and Pathological Correlations. Hong Kong: Mosby-Wolfe; 1993. pp. 6-19.

6. Bordini J, Contar CM, Sarot JR, et al. Multiple compound odontomas in the jaw: case report and analysis of the literature. J Oral Maxillofac Surg 2008;66(12):2617-2620. DOI: 10.1016/j.joms.2007.08.027.

7. Hisatomi M, Asaumi J, Konouchi H, et al. A case of complex odontoma associated with an impacted lower deciduous second molar and analysis of the 107 odontomas. Oral Dis 2002;8(2):100-105. DOI: 10.1034/j.1601-0825.2002.1c778.x.

8. Amado-Cuesta S, Gargallo-Albiol J, Berini-Aytés L, et al. Review of 61 cases of odontoma. Presentation of an erupted complex odontoma. Med Oral 2000;8(5):366-373. 